\title{
NUT midline carcinomas and their differentials by a single molecular profiling method: a new promising diagnostic strategy illustrated by a case report
}

\author{
Simon Haefliger ${ }^{1}$ - Alexandar Tzankov ${ }^{1} \cdot$ Stephan Frank $^{1} \cdot$ Michel Bihl $^{1}$. \\ Alfonso Vallejo ${ }^{1}$. Juerg Stebler ${ }^{2}$. Juergen Hench ${ }^{1}$ (i) \\ Received: 15 April 2020 / Revised: 3 June 2020 / Accepted: 10 June 2020 / Published online: 25 June 2020 \\ (C) The Author(s) 2020
}

\begin{abstract}
NUT midline carcinoma is an aggressive neoplasm defined by chromosomal rearrangements of the nuclear protein in testis (NUT) gene (NUTM1). In this article, we present a strategy to detect this rare tumor through a standard DNA methylation array analysis even when occurring in unusual anatomic sites. We illustrate our approach through a case study in which we detected metastatic spread of a NUT midline carcinoma within a bone marrow biopsy that exhibited histological features of a blastoid, undifferentiated neoplasm. Our strategy builds on molecular data derived from The Cancer Genome Atlas and Gene Expression Omnibus as well as computational strategies adopted from the Brain Tumor Methylation Classifier. It is a combined approach that detects the unusual cell lineage of NUT midline carcinomas and makes diagnostic use of the entity-specific copy number alterations.
\end{abstract}

Keywords NUT midline carcinoma $\cdot$ DNA methylation profile $\cdot$ Copy number variation profile $\cdot$ Machine learning

\section{Introduction}

NUT midline carcinoma (NMC) is an aggressive neoplasm defined by chromosomal rearrangements of the nuclear protein in testis (NUT) gene (NUTM1), which most commonly is fused with genes of the bromodomain and extra-terminal domain family, like bromodomain-containing protein 4 (BRD4) and bromodomain-containing protein 3 (BRD3) [1, 2]. NMC is characterized by a very poor prognosis with a median survival time of 6.7 months and can occur anywhere along the trunk, most commonly along the midline, with typical sites

Simon Haefliger and Alexandar Tzankov contributed equally to this work.

Electronic supplementary material The online version of this article (https://doi.org/10.1007/s00428-020-02869-7) contains supplementary material, which is available to authorized users.

Juergen Hench

jurgen.hench@gmail.com

1 Institute of Pathology, University Hospital Basel, Schönbeinstrasse 40, 4031 Basel, Switzerland

2 Department of Medicine, Cantonal Hospital Baden, Baden, Switzerland being the head, neck, and mediastinum [2, 3]. Classical methods to diagnose NMC rely on morphology, immunohistochemistry, and in situ hybridization. Here, we present a novel strategy to detect this rare tumor directly through a standard DNA methylation array analysis. We illustrate our approach through a case study of a NUT midline carcinoma within a bone marrow biopsy, exhibiting histological features of a blastoid, undifferentiated neoplasm.

\section{Materials and methods}

\section{Illustrative case}

A 71-year-old male presented with B-symptoms (night sweats for the last 6 months and weight loss of $10 \mathrm{~kg}$ within the last 3 months) and lower back pain lasting for more than 6 months. Magnetic resonance imaging (MRI) revealed multiple lesions in the lungs, mediastinum, kidney, liver, and bones. An externally performed biopsy of the pulmonary mass failed to render a conclusive diagnosis. Subsequently, suspecting a hematolymphoid neoplasm because of accompanying cytopenia, a BM biopsy was performed and showed a diffuse 
infiltration by cohesive, blastoid appearing medium- to largesized tumor cells with high mitotic activity (Fig. 1a-c). No squamous or glandular differentiation was found. Suspected differential diagnoses included, among others, melanoma, carcinoma, or (pleomorphic) rhabdomyosarcoma. On immunohistochemistry, the neoplastic cells were positive for CD99 and p63 (Fig. 1d), while remaining negative for ALK, CD3, CD5, CD11c, CD20, CD34, CD79a, CD57, CD117, chromogranin, CK19, CK22, desmin, ERG, Melan-A, HMB45, mast cell tryptase, MPO, MyoD1, PSA, PSAP, S100, TLE1, and WT1 and showed a retained expression of INI-1. Ewing's sarcoma, mesenchymal chondrosarcoma, and NMC were then considered as differentials. Immunohistochemical NUT protein staining showed granular positivity for NUT in more than $50 \%$ of the tumor cells (Fig. 1e). Subsequent fluorescence in situ hybridization (FISH) detected a classical NUTM1 gene break by (Fig. 1f). The patient died 2 months after receiving the diagnosis of NMC.

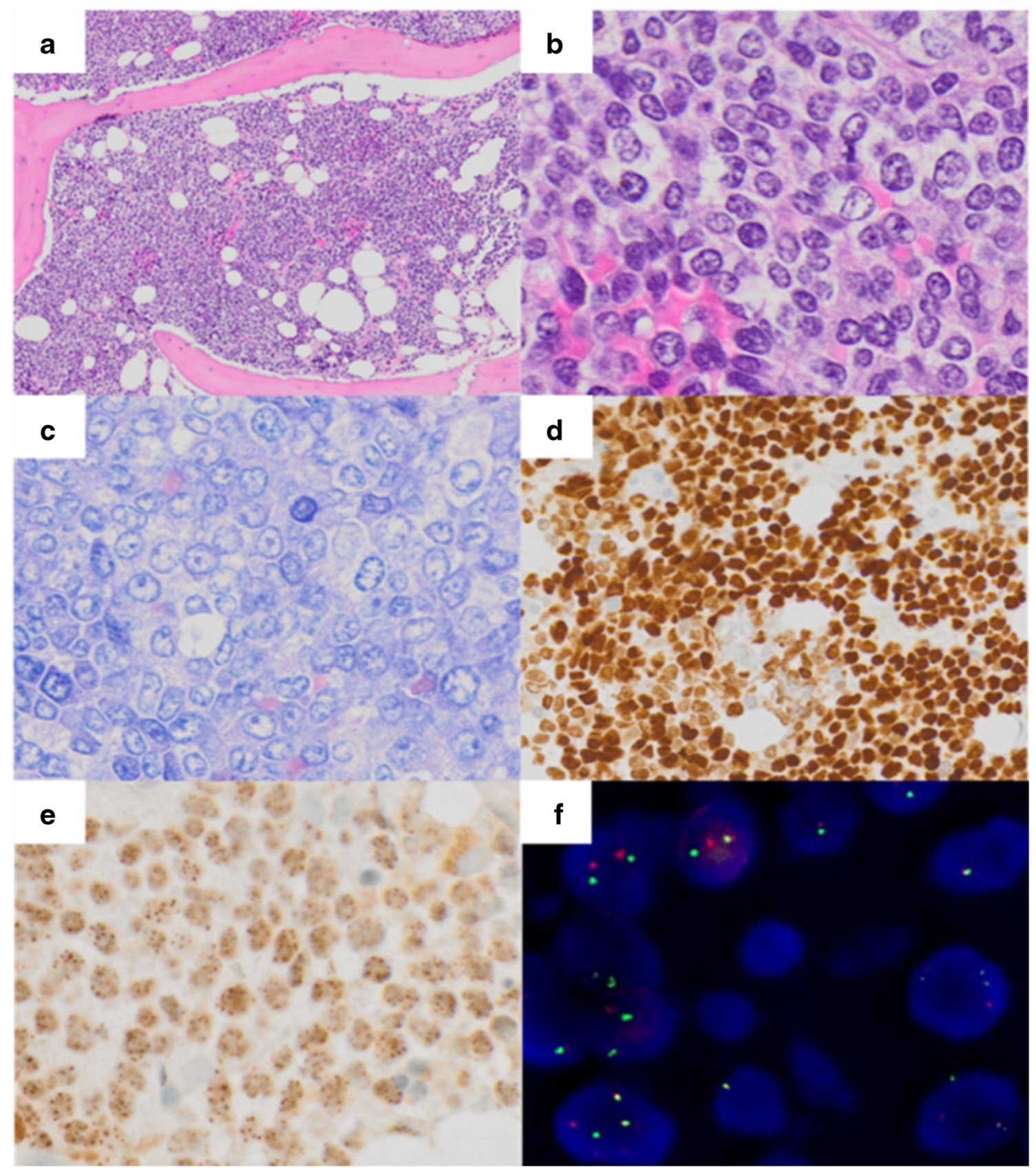

Fig. 1 A-E Sheet-like proliferation of monomorphic cells infiltrating the bone marrow $(\mathbf{a}, \times 10$, Hematoxylin and eosin $(\mathrm{HE}))$. Neoplastic cells feature a blastoid morphology with moderate amounts of clear cytoplasm and round to oval nuclei with vesicular chromatin $(\mathbf{b}, \times 40, \mathrm{HE}$ and $\mathbf{c}, \times$

40, Giemsa), staining for p63 (d, $\times 20$, immunoperoxidase $)$ and NUT (e, $\times 40$, immunoperoxidase). $f$ Dual-color FISH reveals a split-apart of the translocated NUTM1 gene 


\section{DNA methylation array-based computational diag- nostic approach}

Upon request of the treating oncologists, a DNA methylation array was performed. Approximately $1 \mu \mathrm{g}$ of DNA, determined by absorption (Nanodrop method) and representing a tumor area of approximately $0.5 \mathrm{~mm}^{2}$, was used for the analysis. The tumor cell content was $90 \%$. The DNA methylation profile was then obtained on an Infinium Human Methylation EPIC BeadChip array (Illumina, USA). The resulting IDAT files were processed using the in-house diagnostic toolchain EpiDiP (publicly available at http://www.epidip.org) that allows comparison of a given dataset against currently more than 15,500 reference datasets mostly derived from The Cancer Genome Atlas (TCGA) and Gene Expression Omnibus (GEO). In addition, raw data of diagnostically worked up cases from within our institution and collaborators have been injected into this data lake. The analysis considers both the DNA methylation profile and the copy number variations. This software is primarily written in $\mathrm{R}$ (version 3 . 6.2 as of the time of writing this manuscript) and solely relies on open-source packages. IDAT data, the format provided by Illumina Methylation Array scanners, are parsed through minfi (available from Bioconductor) and normalized using the SWAN algorithm (Bioconductor). Since most reference datasets are from the $450 \mathrm{~K}$ methylation array era, we convert all datasets, both from $850 \mathrm{~K} / \mathrm{EPIC}$ arrays as well as $450 \mathrm{~K}$ arrays to an approximately $400 \mathrm{~K}$ probe set present in both arrays. Sex chromosomes and cross-reactive probes [4] are excluded. Filtering for the top differentially methylated probes is performed through a standard deviation of all probes across all datasets. Only the top 25,000 probes of this ranking, adopted from the data preparation method used to build the brain tumor methylation classifier, are considered for dimension reduction by uniform manifold approximation and projection (UMAP, R implementation, available from CRAN) [5]. Specimens sharing epigenomic and hence lineage similarities cluster together as demonstrated previously with t-distributed stochastic neighbor embedding (t-SNE) [6].

Genome-wide copy number alterations are read out through conumee (available on Bioconductor) [7]. Copy number plots are calculated for all cases in the reference data lake and made accessible for each case through EpiDiP.

The pan-data-lake UMAP plot is overlaid with tissue type annotations curated in an in-house database and linked to copy number plots for each sample. These data are presented to the pathologist through a Shiny-based web application that we made available for free online [8] (http://www.epidip.org).

Upon highlighting a particular diagnostic case through searching for its identifier, neighboring cases can be interrogated for their original annotation and their copy number plots can visually be compared. A user-defined set of genomic loci, typically proto-oncogenes and tumor suppressor genes, is annotated through conumee for improved legibility (Fig. 2a).

\section{Results}

Dimension reduction by UMAP indicated a high similarity of the tumor of our illustrative case to squamous carcinomas of the lung (Fig. 2c, d) when compared to over 15,500 reference methylome datasets comprising tumors of diverse differentiation lineages and cells-of-origin. In addition, the chromosomal copy number profile, extracted by conumee, showed a circumscribed loss of heterozygosity (LOH) on chromosome $15 \mathrm{q}$, adjacent to the NUTM1 locus. The raw array data are available in Supplementary File 1. When invoking the copy number profiles of the five closest neighboring cases, they show the rather disrupted genomes typical of mutagenesisinduced squamous cell cancers (Fig. 3), making this case rather different from common pulmonary squamous cell carcinoma.

\section{Discussion}

The histology of NMC corresponds to an undifferentiated tumor that may or may not exhibit squamous cell carcinoma features [2]. The presence of so-called abrupt foci of keratinization is considered a hallmark of NMC [9]. Another distinctive feature is the cellular monomorphism of the poorly differentiated component, contrasting with the highly atypical, polymorphic tumor cells seen in other dedifferentiated carcinomas [10]. The appearance of NMC overlaps with other poorly differentiated small blue round cell tumors such as Ewing's sarcoma or acute leukemia [11, 12]. Classical diagnostic methods rely on morphology, immunohistochemistry, and FISH. Classical squamous cell carcinomas often have complex karyotypes and a high mutation load presumably caused by long-term exposure to mutagens such as ultraviolet light, tobacco, or alcohol [2]. In this regard, NMC is clearly different, as this tumor is likely driven by its typical single-gene rearrangement as suggested by the otherwise minor cytogenetic aberrations. Indeed, our analyses revealed a circumscribed deletion involving parts of chromosome 15q, adjacent to the NUTM1 locus (Fig. 2a, b), and a NUTM1 break by FISH (Fig. 1f).

Even without any prior knowledge of NUT midline carcinoma, the epigenomic and copy number data from our illustrative case are in line with the hypothesis that such tumors feature a squamous cell-like differentiation and a "flat genome" typical of fusion-driven neoplasms with distinct numeric changes found in the vicinity of a chromosomal break [7]. We are aware that our strategy we routinely apply to a wide range of neoplasms has so far been tested with only a single NNC case; however, we make available our data and analysis platform for public use with the intention to facilitate the collection of further samples of NUT midline carcinoma. The ultimate aim is to include NMC in future machine learning training datasets making it detectable by same-day DNA methylation/CNV diagnostics based on Oxford Nanopore 
a $\quad$ 202123800244 R01C01
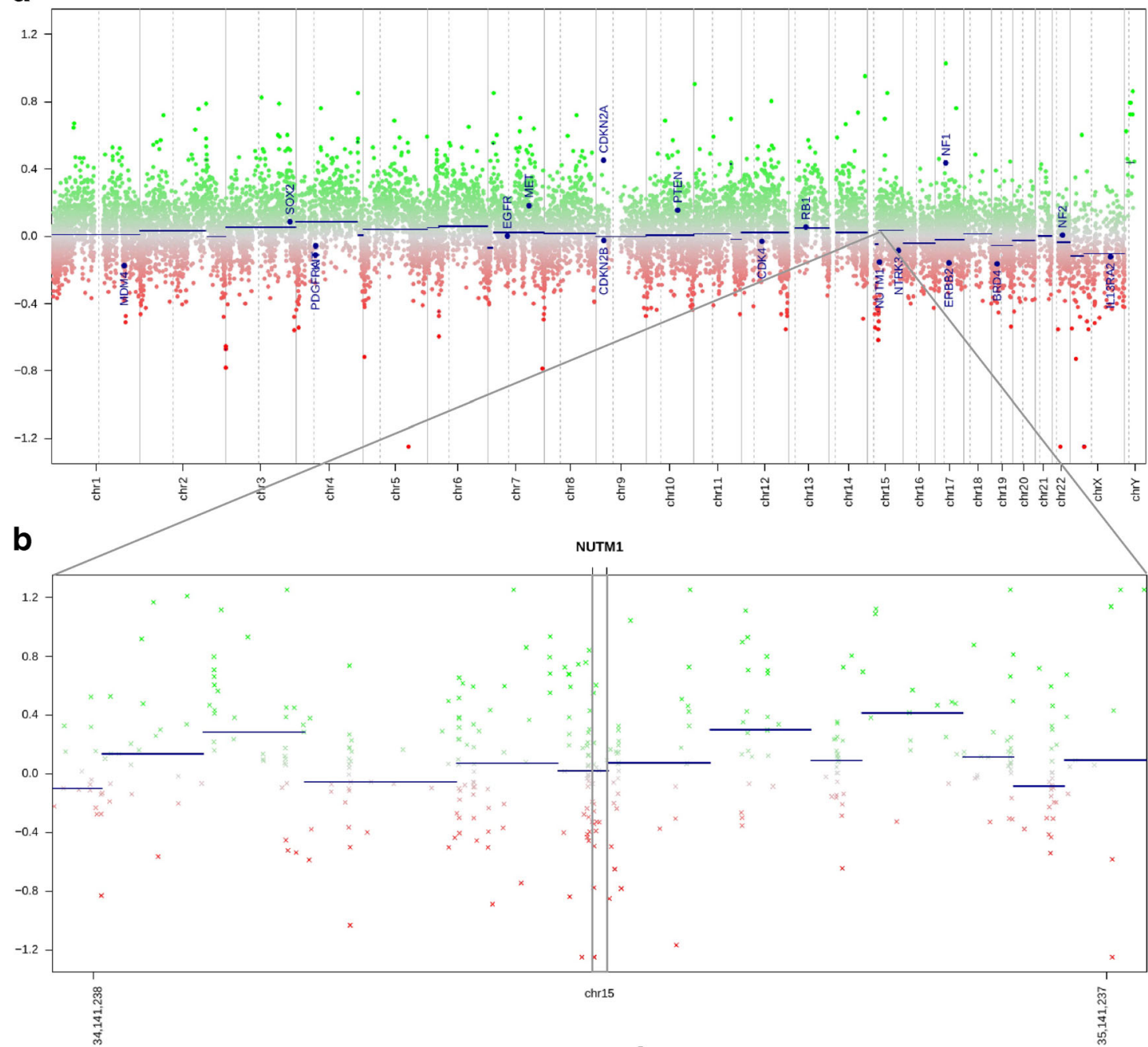

C

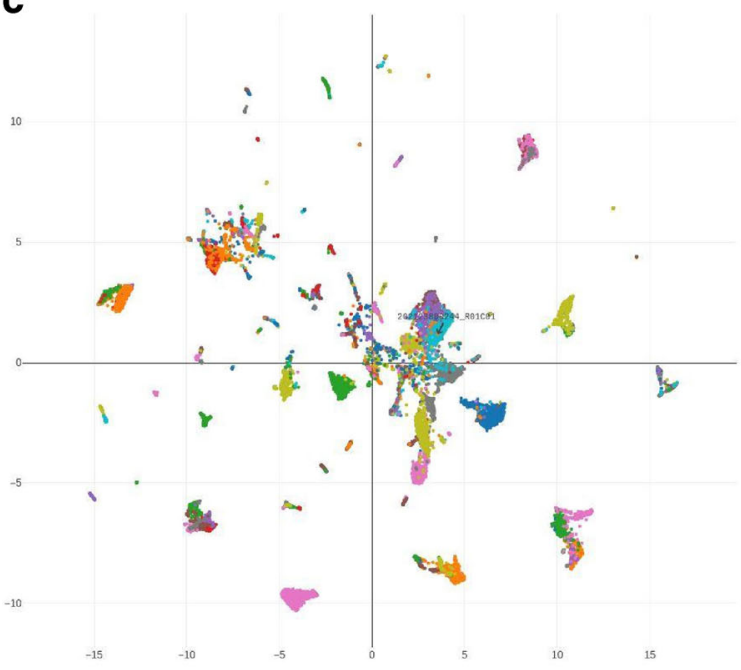

Fig. 2 a Genome-wide copy number plot calculated from microarray data. The level of the blue dot labeled NUTM1 represents the average copy number for all NUTM1 probes. b Magnified plot around and including NUTM1 (center, black box). Note the partial loss (LOH) to the left of the NUTM1 locus, also including some of the NUTM1 probes. c Overview of the UMAP plot for $>15,000$ reference samples; patient sample highlighted by an arrow d

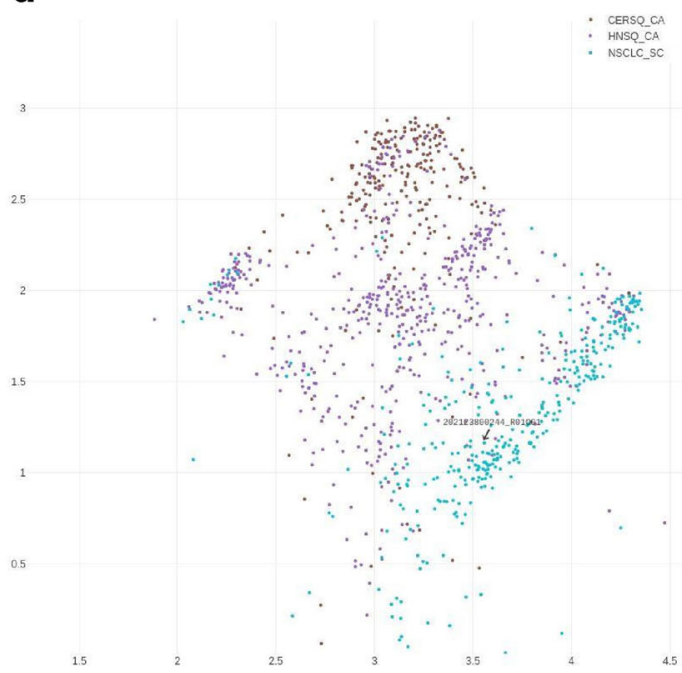

and array ID. This respective cluster of samples mainly comprises carcinomas of various lineages. d Magnification from $\mathbf{c}$. The tumor localizes in a subcluster containing cancers with squamous cell differentiation. Within this cluster, the similarity is highest to squamous cell cancers (SCCs) of the lung. Abbreviations of the color legend for methylation classes: CERSQ CA cervical SCC, HNSQ CA head and neck SCC, NSCLC_SC lung SCC 
Fig. 3 Genome-wide copy number plots calculated from microarray data for the five closest UMAP neighbor cases, all representing squamous cell cancers of the lung. All neighboring cases are TCGA datasets. Note multiple complex copy number alterations in all cases, distinguishing conventional lung squamous cell cancer from the fusion-driven NUT midline carcinoma despite the epigenomic similarities between these entities
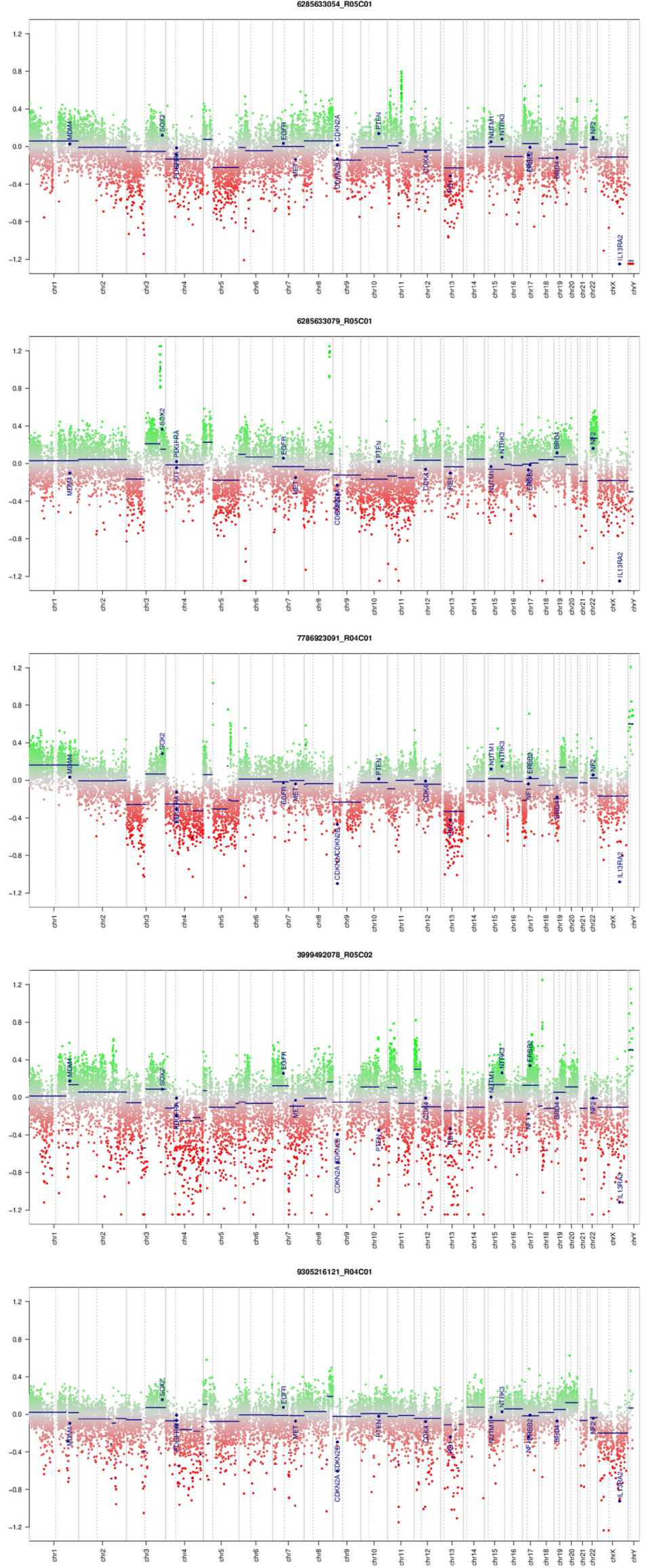
sequencers, too (ONT, UK) [13]. Meanwhile, we propose our strategy to recognize this tumor type based on methylation array data by manual inspection of methylation data through dimension reduction and concurrent copy number profile visualization. Previously published work such as EPICUP [14] would in such cases report "squamous cell cancer of the lung" which is somewhat correct from an epigenomic point of view but does not provide a helpful hint in the given scenario, rather being misleading. EPICUP never provided the pathologist with accompanying $\mathrm{CNV}$ profiles. This diagnostically helpful strategy was originally implemented alongside the Brain Tumor Methylation Classifier [15] but is currently restricted to brain tumors. Hence, we also integrated CNV profiles in our UMAP-based tool.

Methylation analysis readily covers all differential diagnoses brought up in our illustrative case. Our UMAP/CNV tool [8] is, in addition, aware of a broad spectrum of hematolymphoid, epithelial, melanocytic, neuroendocrine, cerebral, and mesenchymal neoplasms all of which are represented through publicly available raw data. In summary, we present an alternative, modern, and timely way to detect NMC by a single microarray-based test.

Acknowledgments We thank Martin Sill (DKFZ) and David Capper (Charité Berlin) for their excellent primer on how to analyze methylation data through $\mathrm{R}$.

Author contributions S.H., A.T., and J. H. conceived and designed the project. J. H., S.F., and M.B. analyzed the molecular data. S.H, A.T., S.F., M.B., A.V., J.S., and J.H. wrote the manuscript. All the authors agreed to the content of the manuscript.

\section{Compliance with ethical standards}

Conflict of interest The authors declare that they have no conflict of interest.

Informed consent Written informed consent was obtained from the patient.

Open Access This article is licensed under a Creative Commons Attribution 4.0 International License, which permits use, sharing, adaptation, distribution and reproduction in any medium or format, as long as you give appropriate credit to the original author(s) and the source, provide a link to the Creative Commons licence, and indicate if changes were made. The images or other third party material in this article are included in the article's Creative Commons licence, unless indicated otherwise in a credit line to the material. If material is not included in the article's Creative Commons licence and your intended use is not permitted by statutory regulation or exceeds the permitted use, you will need to obtain permission directly from the copyright holder. To view a copy of this licence, visit http://creativecommons.org/licenses/by/4.0/.

\section{References}

1. French CA, Ramirez CL, Kolmakova J et al (2008) BRD-NUT oncoproteins: a family of closely related nuclear proteins that block epithelial differentiation and maintain the growth of carcinoma cells. Oncogene 27:2237-2242. https://doi.org/10.1038/sj.onc. 1210852

2. French C (2014) NUT midline carcinoma. Nat Rev Cancer 14:149150. https://doi.org/10.1038/nrc3659

3. Bauer DE, Mitchell CM, Strait KM et al (2012) Clinicopathologic features and long-term outcomes of NUT midline carcinoma. Clin Cancer Res 18:5773-5779. https://doi.org/10.1158/1078-0432. CCR-12-1153

4. Chen Y, Lemire M, Choufani S et al (2013) Discovery of crossreactive probes and polymorphic $\mathrm{CpGs}$ in the Illumina Infinium HumanMethylation450 microarray. Epigenetics 8:203-209. https://doi.org/10.4161/epi.23470

5. McInnes L, Healy J, Melville J (2018) UMAP: uniform manifold approximation and projection for dimension reduction. ArXiv180203426 Cs Stat.

6. van der Maaten L, Hinton G (2008) Visualizing data using t-SNE. J Mach Learn Res 9:2579-2605

7. Capper D, Stichel D, Sahm F et al (2018) Practical implementation of DNA methylation and copy number-based CNS tumor diagnostics: the Heidelberg experience. Acta Neuropathol (Berl) 136:181210. https://doi.org/10.1007/s00401-018-1879-y

8. Hench J, Frank S (2020) EpiDiP Server. http://www.epidip.org

9. Travis WD, World Health Organization, International Agency for Research on Cancer (2015) WHO classification of tumours of lung, pleura, thymus and heart: ... reflects the views of a working group that convened for a consensus and editorial meeting at the International Agency for Research on Cancer, Lyon, April 24-26, 2014, 4. ed. International Agency for Research on Cancer, Lyon.

10. French CA (2018) NUT carcinoma: clinicopathologic features, pathogenesis, and treatment: NUT Carcinoma. Pathol Int 68:583595. https://doi.org/10.1111/pin.12727

11. Mertens F, Wiebe T, Adlercreutz C et al (2007) Successful treatment of a child with $\mathrm{t}(15 ; 19)$-positive tumor: $\mathrm{t}(15 ; 19)$-Positive Tumor. Pediatr Blood Cancer 49:1015-1017. https://doi.org/10. $1002 /$ pbc. 20755

12. Li W, Chastain K (2018) NUT midline carcinoma with leukemic presentation mimicking CD34-positive acute leukemia. Blood 132: 456-456. https://doi.org/10.1182/blood-2017-07-796268

13. Euskirchen P, Bielle F, Labreche K et al (2017) Same-day genomic and epigenomic diagnosis of brain tumors using real-time nanopore sequencing. Acta Neuropathol (Berl) 134:691-703. https://doi.org/ 10.1007/s00401-017-1743-5

14. Moran S, Martínez-Cardús A, Sayols S et al (2016) Epigenetic profiling to classify cancer of unknown primary: a multicentre, retrospective analysis. Lancet Oncol 17:1386-1395

15. Capper D, Jones DTW, Sill M et al (2018) DNA methylation-based classification of central nervous system tumours. Nature 555:469474. https://doi.org/10.1038/nature26000

Publisher's note Springer Nature remains neutral with regard to jurisdictional claims in published maps and institutional affiliations. 